\title{
Plataforma digital do Observatório Socioespacial da Baixada Santista - Observa BS 1
}

\section{Digital platform of the Observatory Socio-Spatial of the Baixada Santista - Observa BS}

\author{
Verde, Letícia Passarelli1; Viana, Mônica A. ${ }^{2}$; Portela, B. R. ${ }^{3}$ \\ 1 Universidade Católica de Santos, UniSantos, Santos, Brasil, \\ leticiapassarelliverde@UniSantos.br \\ 2 Pontífica Universidade Católica de Campinas, PUC Campinas, \\ moviana@UniSantos.br \\ 3 Universidade Católica de Santos, UniSantos, \\ beatriz.ramos.portela@gmail.com
}

\begin{abstract}
RESUMO
O grupo de pesquisa Observatório Socioespacial da Baixada Santista da Universidade Católica de Santos - Observa BS estuda as questões urbanas, com foco na Região Metropolitana da Baixada Santista - RMBS e seus nove municípios. A produção do grupo é de interesse acadêmico e público, mas cabe a sistematização e divulgação desse material. Neste sentido, as pesquisas de Iniciação Científica, com apoio da bolsa PIBIC-CNPq, estruturaram a modelagem de uma plataforma digital que visa subsidiar pesquisas, projetos de extensão e as disciplinas da graduação e pós-graduação. Contribuir para monitorar as dinâmicas socioespaciais e políticas públicas de desenvolvimento urbano e regional, na integração entre ensino, pesquisa e extensão na Universidade e no fortalecimento da participação e controle social. O desenvolvimento se deu de forma inovadora com a integração e interdisciplinaridade entre os cursos de Arquitetura e Urbanismo e Ciências da Computação. A metodologia baseou-se em pesquisas bibliográfica e documental, levantamento de plataformas referenciais, sistematização e organização de dados. Esperase que a plataforma digital do Observa BS possa contribuir para a democratização de dados e informações, na construção de indicadores e no monitoramento e análises críticas das dinâmicas socioespaciais na RMBS, considerando que é um processo contínuo, mas com resultados concretos.
\end{abstract}

Palavras-chave: Baixada Santista, Banco de dados, Plataforma digital, Observa BS, Planejamento Urbano e Regional.

\section{ABSTRACT}

The Observatory Socio-spatial of the Baixada Santista of the Catholic University of Santos Observa BS, is a research group that studies the urban issues, focusing on the Metropolitan

\footnotetext{
${ }^{1}$ VERDE, Letícia Passarelli; VIANA, Mônica A.; PORTELA, B. R. Plataforma Digital do Observatório Socioespacial da Baixada Santista - OBSERVA BS. In: II SIMPÓSIO NACIONAL DE GESTÃO E ENGENHARIA URBANA: SINGEURB, 2019, São Paulo. Anais... Porto Alegre: ANTAC, 2019.
} 
Region of Baixada Santista - RMBS and its nine municipalities. The group production is academic and public interest, but needs the systematization and dissemination of this material. That way, the research of Scientific Initiation with the support of PIBIC CNPq scholarship, structured the modeling of a digital data platform that aims to support research, extension projects of undergraduate and postgraduate subjects. In addition, contribute to monitor the socio-spatial dynamics and public policies of urban and regional development, the integration among teaching, research and extension in the University and the strengthening of participation and social control. The research development took place in an innovative way with the integration and interdisciplinarity among the courses Architecture and Urbanism and Computer Science. The methodology included bibliographic and desk research, survey of reference platforms, systematization and data organization. It is expected that the Observa BS digital platform will contribute to the data and information democratization, the construction of indicators, monitoring and critical analysis of the socio-spatial dynamics of RMBS, whereas the continuous process, but with concrete results.

Keywords: Baixada Santista, Database, Digital Platform, Observa BS, Urban and Regional Planning.

\section{INTRODUÇÃO}

Considerando a pesquisa como uma atividade constante de coleta de informações de maneira organizada e sistematizada, a plataforma digital do Observa BS visa disponibilizar dados e informações sobre questões urbanas e metropolitanas já existentes e também produzidas pelo grupo de pesquisa, possibilitando o acesso à informação qualificada e contribuindo com pesquisas, avaliação e monitoramento das dinâmicas socioespaciais na RMBS e seus municípios, além de possibilitar a criação de indicadores sociais. Segundo Jannuzzi (2002) esses indicadores auxiliam no planejamento público mediante a uma avaliação mais consistente quanto à concepção e análise de políticas públicas em diversos âmbitos², como também no monitoramento pelo poder público e a sociedade civil.

Em um mundo dinâmico, acrescido constantemente de novas descobertas, de partilhas intensas, percepções discrepantes, posições divergentes e ações contraditórias, cada um obriga-se a buscar novas explicações e alcançar uma compreensão mais abrangente tanto dos fatos como dos atos quanto das realidades individual e social nas quais cada um está vivendo. (CHIZZOTTI, 2001, p. 107)

Neste contexto, o Observa BS vem desenvolvendo sua plataforma digital a partir de um banco de dados para disponibilizar essas informações. Um banco de dados, de acordo com Rezende (2006) e o web site CCM (2017), consiste no armazenamento de dados de maneira estruturada, o chamado back, e sua utilidade se dá pela disposição dos mesmos para consulta, introdução ou atualização, e o front, é como a plataforma se apresenta ao internauta e o conteúdo que ele tem acesso.

\section{OBJETIVOS}

O objetivo do trabalho é expor o processo de concepção e elaboração da Plataforma Digital do Observatório Socioespacial da Baixada Santista da Universidade Católica de Santo, sobre - processo de produção e transformação do espaço urbano-regional da Região Metropolitana da Baixada Santista - RMBS. A partir dessa apresentação, colocar em debate tanto o processo de elaboração da plataforma digital, quanto seus resultados e prognósticos dos impactos desse instrumento de pesquisa, buscando um intercâmbio de experiências, a fim de somar esforços para qualificação da Plataforma Digital do Observa BS.

\footnotetext{
${ }^{2}$ Tais como para a elaboração de planos diretores de desenvolvimento urbano, avaliação de impactos ambientais por grandes empreendimentos, justificativa de repasses de verbas públicas para programas sociais e atendimento à necessidade de disponibilização de equipamentos ou serviços sociais para públicos específicos.
} 


\section{METODOLOGIA}

Com a proposta de integração entre cursos, houve a divisão de tarefas: para área de Arquitetura e Urbanismo coube levantar as referências teóricas e empíricas de plataformas digitais, subsidiando a conceituação, modelagem e estruturação da plataforma digital do Observa BS, em sequência a sistematização, coleta e organização de informações; já para Ciências da Computação coube a parte operacional da modelagem da plataforma digital.

A partir dos objetivos estabelecidos no projeto de pesquisa e no grupo Observa BS, a metodologia da pesquisa científica se desenvolveu seguindo as premissas de se somar aos processos de monitoramento existentes da região, tais como a plataforma de indicadores do Observatório Litoral Sustentável do Instituto Pólis, o Sistema de Monitoramento e Avaliação SIMA da Agência Metropolitana da Baixada Santista (AGEM-BS) e o SigSantos da Prefeitura de Santos, contando com o apoio de instituições parceiras como o Instituto Polis, a Unifesp e a AGEM-BS.

\section{DESENVOLVIMENTO}

Com base nas plataformas referenciais pesquisadas, foram verificados os aspectos como a estética, classificada com boa, regular ou ruim, relacionadas à polvição visual; se a plataforma é intuitiva, ou seja, de fácil acesso às informações de maneira objetiva e com menor número de redirecionamentos possíveis; os aspectos negativos; e por fim os aspectos positivos que poderiam ser apropriados na modelagem da plataforma. A análise é verificada conforme "Quadro 1" abaixo: 
Quadro 1 - Plataformas Referenciais

\begin{tabular}{|c|c|c|c|c|}
\hline $\begin{array}{l}\text { Plataforma } \\
\text { (Hiperlink) }\end{array}$ & Estética & Intuitivo & Aspectos Negativos & Aspectos Positivos \\
\hline $\begin{array}{c}\frac{\text { Núcleo de }}{\text { Estudos de }} \\
\text { População "Elza } \\
\text { Bercuó" } \\
\text { (NEPO/Unicamp) }\end{array}$ & Boa & Regular & $\begin{array}{l}\text { Público restrito, voltado } \\
\text { à pesquisadores }\end{array}$ & $\begin{array}{c}\text { Apresentação na página } \\
\text { inicial; Integração } \\
\text { biblioteca da Unicamp; } \\
\text { Opção "links interessantes" }\end{array}$ \\
\hline $\begin{array}{l}\frac{\text { Programa }}{\text { Cidades }} \\
\text { Sustentáveis }\end{array}$ & Regular & Não & $\begin{array}{c}\text { Informações } \\
\text { polarizadas; Biblioteca } \\
\text { sem padrão de formato } \\
\text { para download }\end{array}$ & $\begin{array}{l}\text { Estruturação por eixos; } \\
\text { Indicadores; Agendas }\end{array}$ \\
\hline $\begin{array}{l}\frac{\text { Agência }}{\text { Metropolitana da }} \\
\frac{\text { Baixada Santista }}{\text { (AGEM-BS) }}\end{array}$ & Boa & Regular & $\begin{array}{l}\text { Opções de download } \\
\text { descentralizadas }\end{array}$ & $\begin{array}{l}\text { Apresentação fotográfica } \\
\text { slide dos municípios; Menu }\end{array}$ \\
\hline$\frac{\text { GeopSampa }}{\text { (PMSP) }}$ & Boa & Sim & - & $\begin{array}{c}\text { Mapa interativo; } \\
\text { sobreposição de layers; } \\
\text { Download em imagem; } \\
\text { Compatível com Qgis } \\
\text { (Shapefile); Preciso; } \\
\text { Atualização frequente }\end{array}$ \\
\hline$\frac{\text { Santos Mapeada }}{\text { (SigSantos) (PMS) }}$ & Regular & Regular & $\begin{array}{c}\text { Baixa frequência } \\
\text { atualização; Poucas } \\
\text { informações mapeadas; } \\
\text { Baixa precisão; Sem } \\
\text { opção de download } \\
\text { para o mapa, apenas } \\
\text { dados }\end{array}$ & $\begin{array}{l}\text { Noção geral do } \\
\text { mapeamento; Dados em } \\
\text { gráficos }\end{array}$ \\
\hline$\frac{\frac{\text { Prefeitura }}{\text { Municipal de }}}{\underline{\text { Santos }}}$ & Boa & Não & $\begin{array}{c}\text { Excessos de informações } \\
\text { na página inicial; } \\
\text { Legislação Urbanística } \\
\text { "escondida" }\end{array}$ & $\begin{array}{l}\text { Atualização frequente; } \\
\text { Download arquivos; Eixos } \\
\text { temáticos }\end{array}$ \\
\hline RioNow & Boa & Boa & - & $\begin{array}{l}\text { Iniciativa Iniciação } \\
\text { Científica; Dados e } \\
\text { informações em recorte de } \\
\text { tempo (2009-2016/Copa e } \\
\text { Olimpíadas); Download de } \\
\text { arquivos }\end{array}$ \\
\hline Seu Acervo & Boa & Boa & - & $\begin{array}{l}\text { Visualização e download } \\
\text { de Trabalhos Finais de } \\
\text { Graduação (Arquitetura e } \\
\text { Urbanismo) }\end{array}$ \\
\hline
\end{tabular}

Fonte: Letícia Passarelli Verde, 2019 
O Instituto Polis foi a principal referência por ser parceiro da UniSantos, por um termo de cooperação técnica e científica. Foi realizada uma visita técnica e reunião para a compreensão da Plataforma de Indicadores de Monitoramento - Observatório Litoral Sustentável, que atualmente se encontra fora do ar devido ao encerramento do contrato de patrocínio. Com base nessa experiência e no Plano de Ação do Observa BS foi criado um roteiro de sistematização (Figura 1), coletados dados e informações elaborados pelos integrantes do grupo de pesquisa.

Figura 1 - Sistematização e formatos dos arquivos a serem disponibilizados

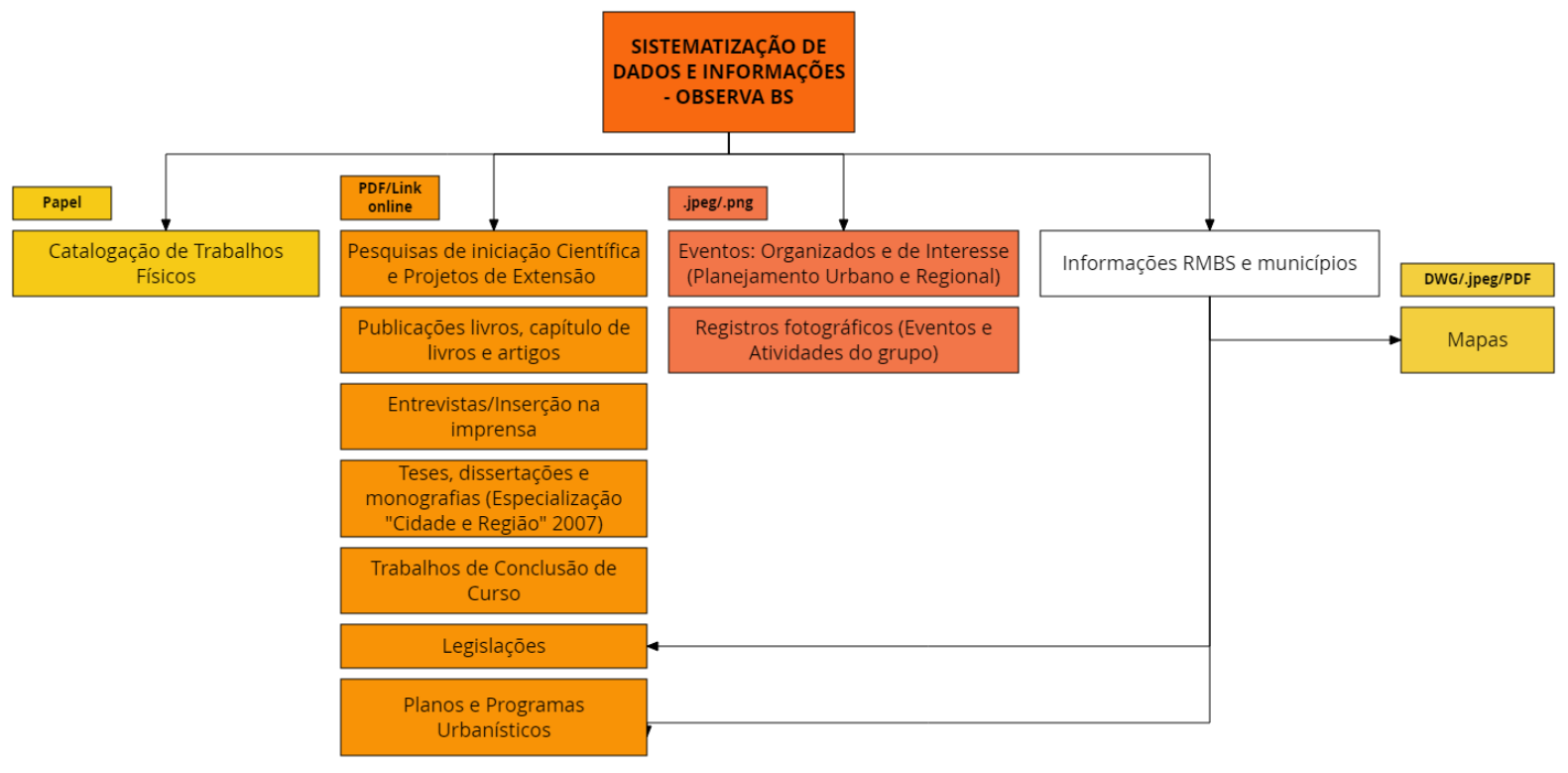

Fonte: Letícia Passarelli Verde(2019)

Após essa sistematização foi criado um drive para o armazenamento das informações em pastas para posterior interface com a plataforma digital. Foi então, iniciada a modelagem da plataforma digital do Observa BS. Para otimização da sistematização dos arquivos a serem disponibilizados, houve o auxílio das bibliotecárias da Unisantos através de uma oficina sobre catalogação e codificação de Arquivos, livros, dados e afins.

\section{RESULTADOS}

Com base nos objetivos e metodologia apresentados, a plataforma digital do Observa BS se desenvolveu da seguinte forma:

Figura 2 - Processo de elaboração da plataforma digital do Observa BS

Organização, sistematização e estruturação da plataforma dados e informações do Observatório Socioespacial da Baixada Santista da UniSantos - Observa BS
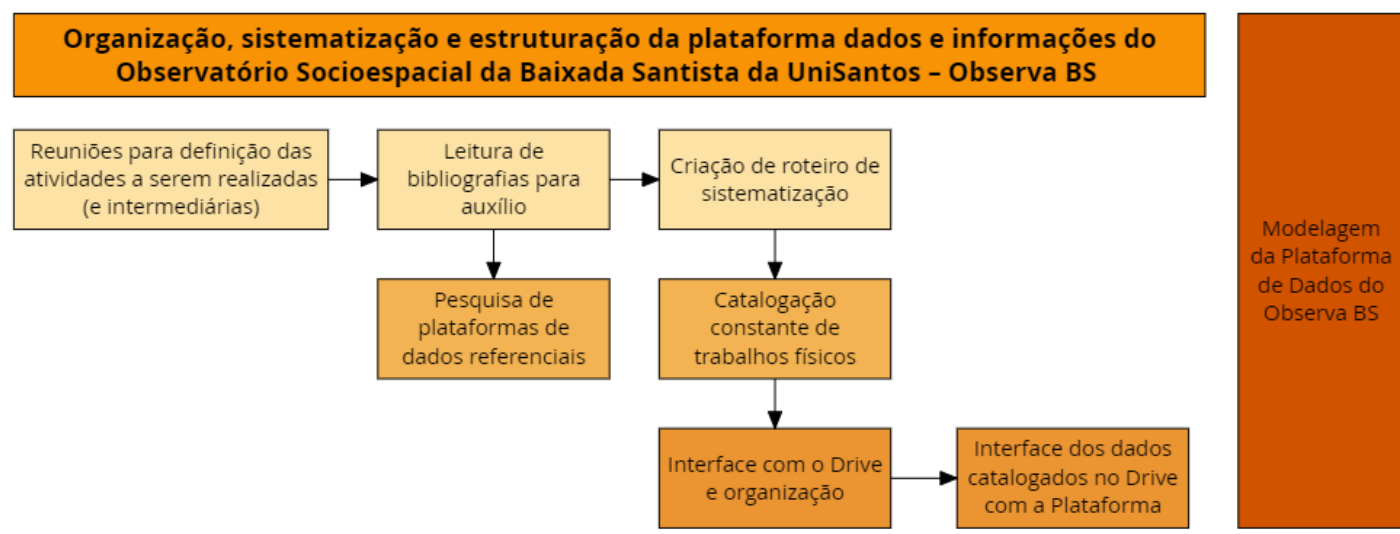

Fonte: Letícia Passarelli Verde(2018) 
Após a organização e sistematização dos dados elaborados pelos integrantes do Observa BS, docentes e discentes, a plataforma foi estruturada em quatro grandes eixos temáticos que sustentam a área de atuação e investigação do grupo de pesquisa Observa BS (Figura 3).

Figura 3 - Eixos estruturantes da plataforma digital de Dados e Informações do Observa BS

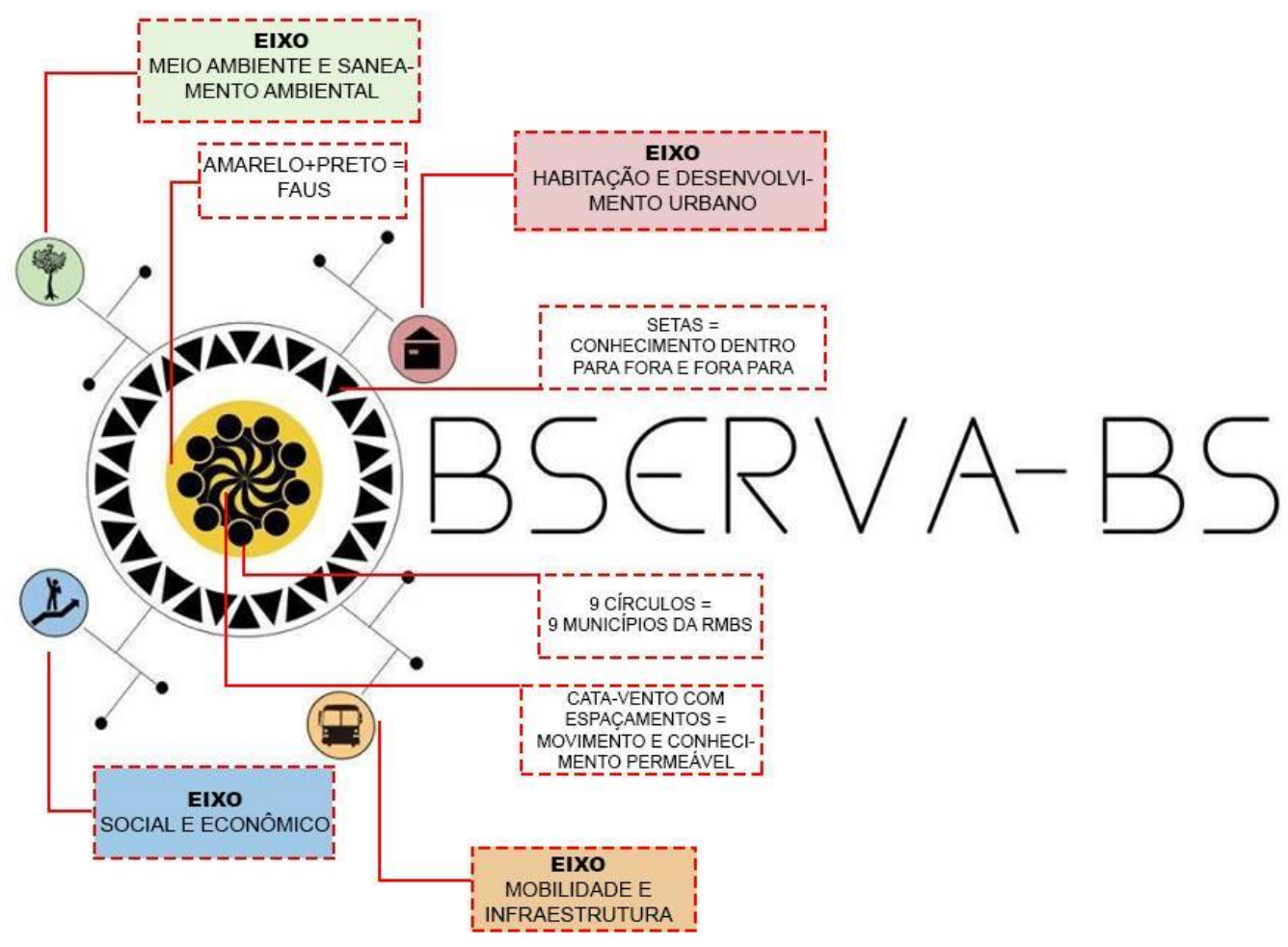

Fonte: Logo por Tainá Muniz (2016); Análise sobre logo por Letícia Passarelli Verde(2018)

A modelagem do front foi desenvolvida em conjunto, entre os alunos e professores dos dois cursos, procurando atender de forma objetiva e acessível dos conteúdos a serem disponibilizados ao usuário e ao internauta, fazendo os ajustes necessários para alcançar esses objetivos.

Inicialmente foi definida a paleta de cores (Figura 4) da plataforma digital com cores mais claras e leves, a fim de não gerar algum incômodo ao usuário.

Figura 4 - Paleta de cores para plataforma digital

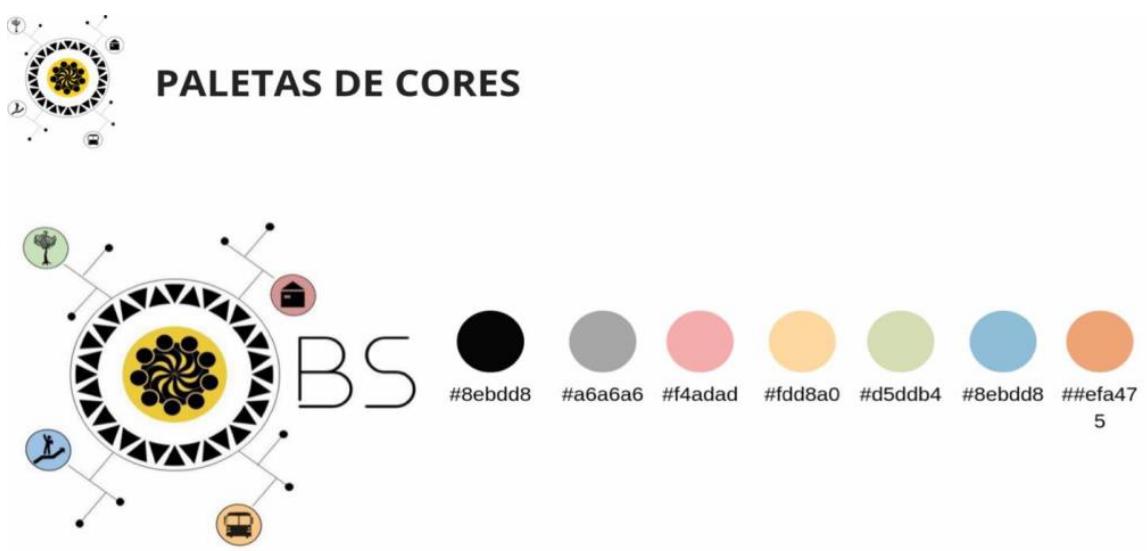

Fonte: Beatriz Ramos Portela (2019) 
A página inicial conta com uma barra de menu sistematizada em: estática na parte superior e inferior, garantindo que o internauta nunca se perca, onde na parte superior há as opções de "Quem somos", "RMBS", "Municípios", "Legislação", "Mídias" e "Eventos", e na parte inferior as opções de "Contato" , "Conheça nossos parceiros" e direcionamentos para as redes sociais como página do Facebook do Observa BS e Portal da Unisantos; e dinâmico em sua parte central, o internauta poderá selecionar o município da RMBS desejado, onde visualizará uma pequena introdução sobre o município, acesso aos mapas disponíveis em diferentes formatos e visualizará os eixos estruturantes e legislação (Figura 8), e ao selecionar um eixo terá acesso aos dados e informações relacionados ao mesmo, caso o usuário selecione RMBS como um todo, seguirá a mesma estrutura, mas acrescido da opção de "Pesquisa", podendo fazer uma pesquisa mais direcionada sobre o que desejar.

Figura 5 - Página inicial da plataforma - Diagrama identificando as partes estáticas e dinâmicas da Plataforma.

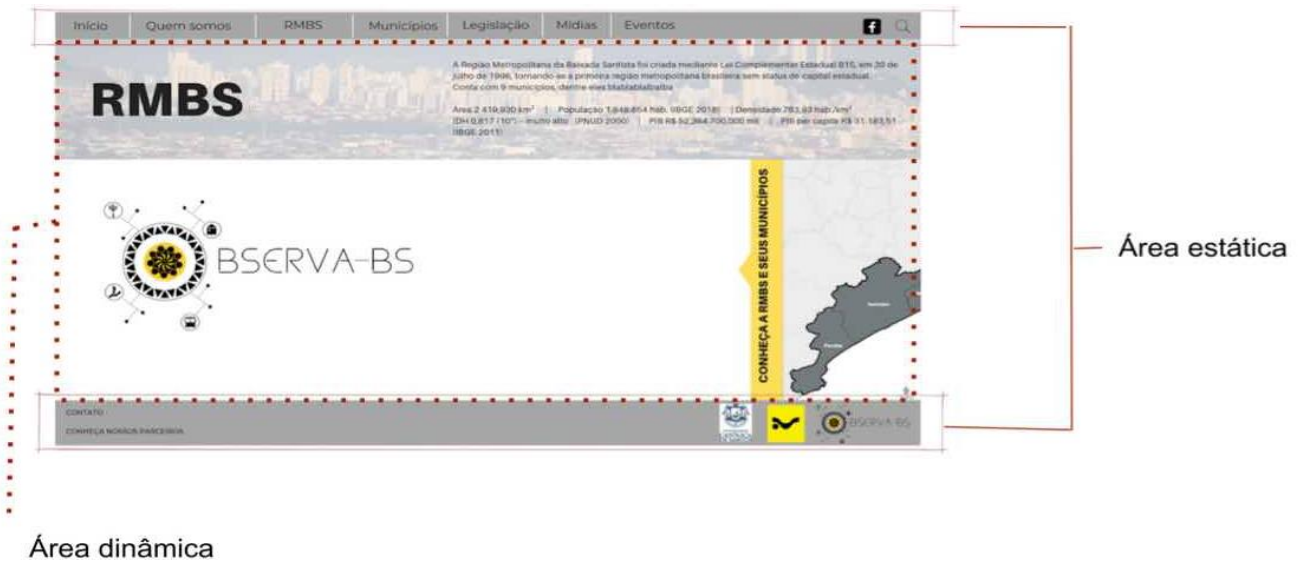

Fonte: Beatriz Ramos Portela (2019).

Figura 6 - Desenvolvimento dos estudos para front da plataforma

Imagens da Plataforma no estudo inicial do front para plataforma digital

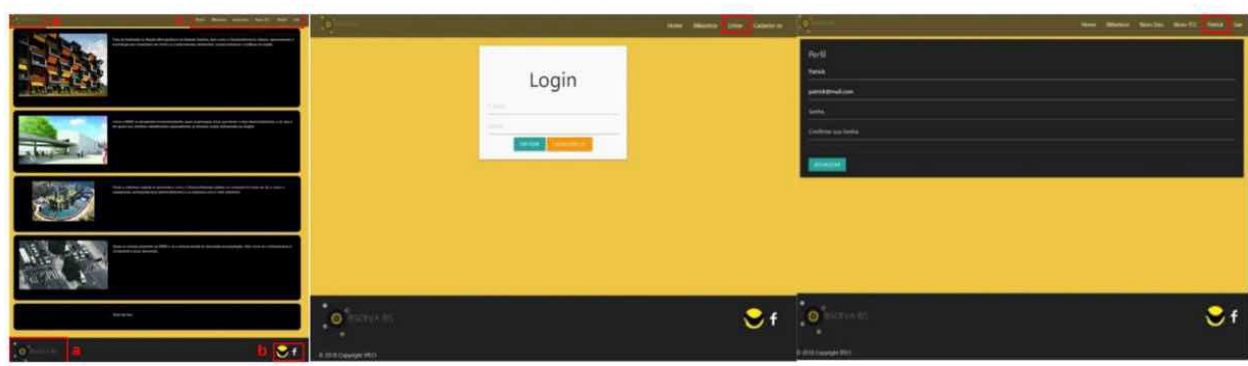

Imagens do estudo final do front para Plataforma digital

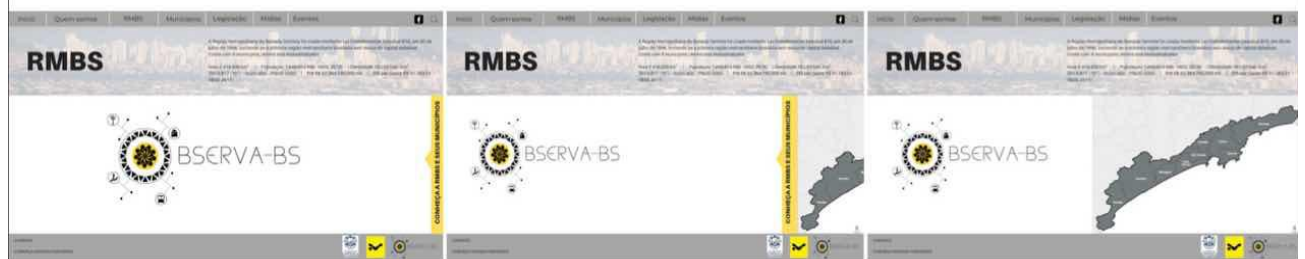

Fonte: Patrick Albino de Lucca (2018); Beatriz Ramos Portela (2019) 
Figura 7 - Imagens da nova front para a plataforma
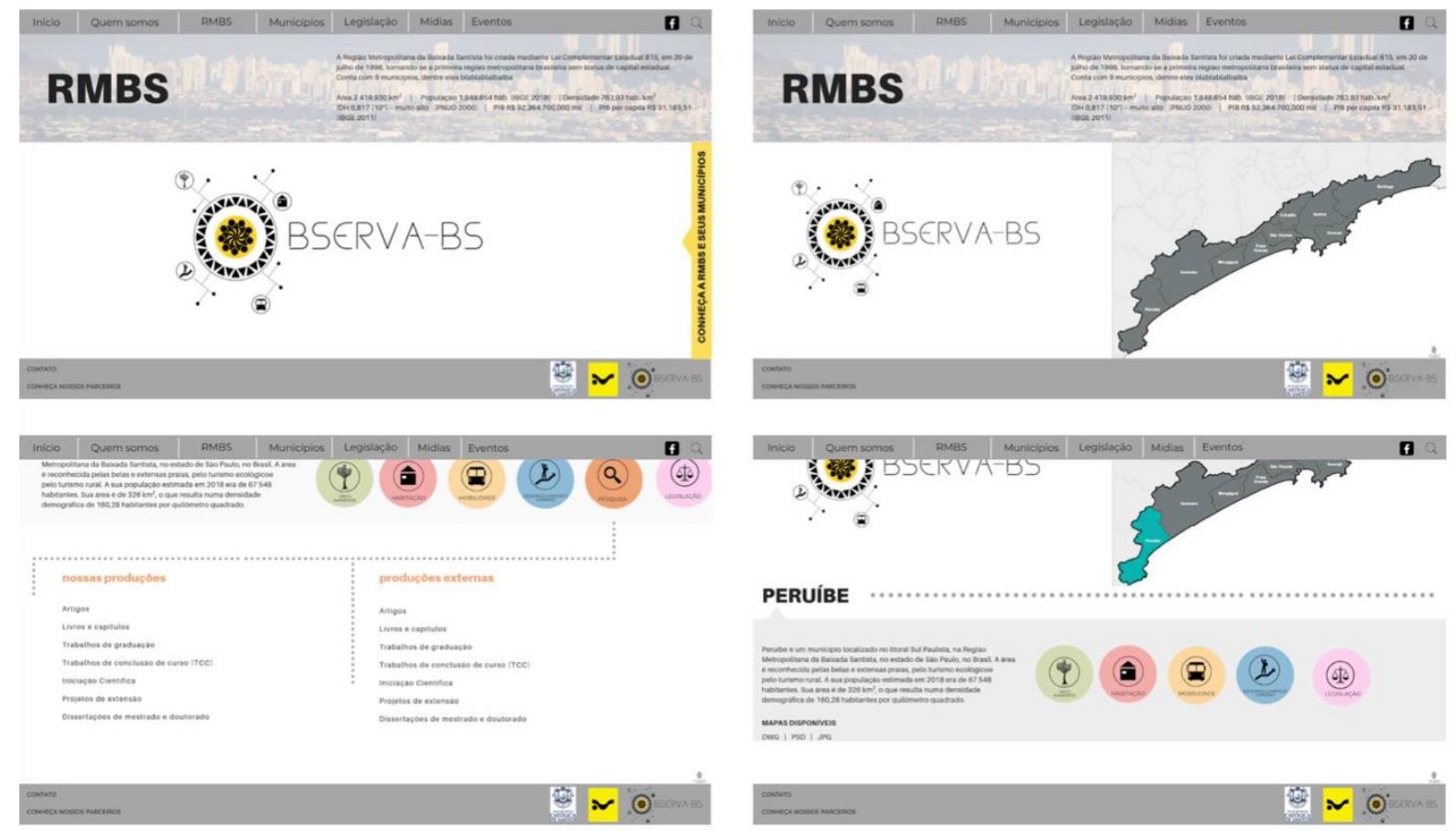

Fonte: Beatriz Ramos Portela (2019)

Figura 8 - Seleção de um município: como os eixos se apresentam

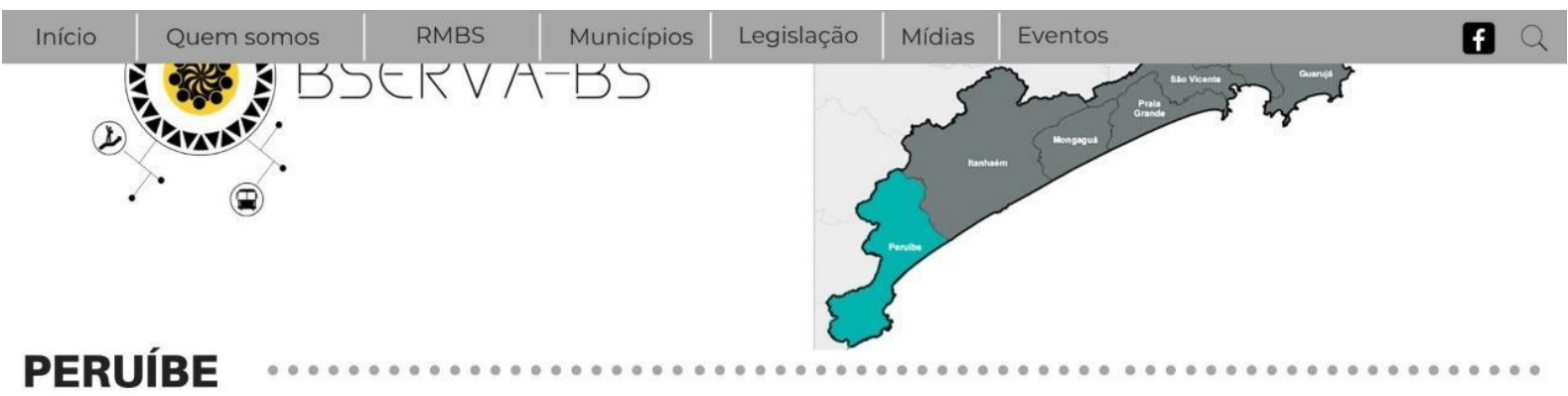

Peruibe é um municipio localizado no litoral Sul Paullsta, na Regiào Metropolitana da Baixada Santista, no estado de Sảo Paulo, no Brasil. A área é reconhecida pelas belas e extensas praias, pelo turismo ecologicoe pelo turismo rural. A sua populaçấo estimada em 2018 era de 6754 habitantes. Sua área è de $326 \mathrm{~km}^{2}$, o que resulta numa densida

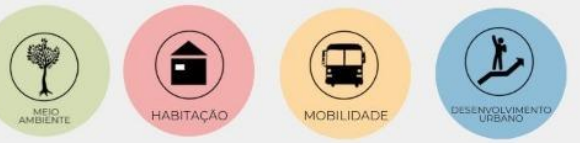


A fim de sintetizar o processo de disponibilização por meio da plataforma digital do Observa BS, trabalhará da seguinte forma:

Figura 9 - Forma de funcionamento da Plataforma de Dados e Informações do Observa BS

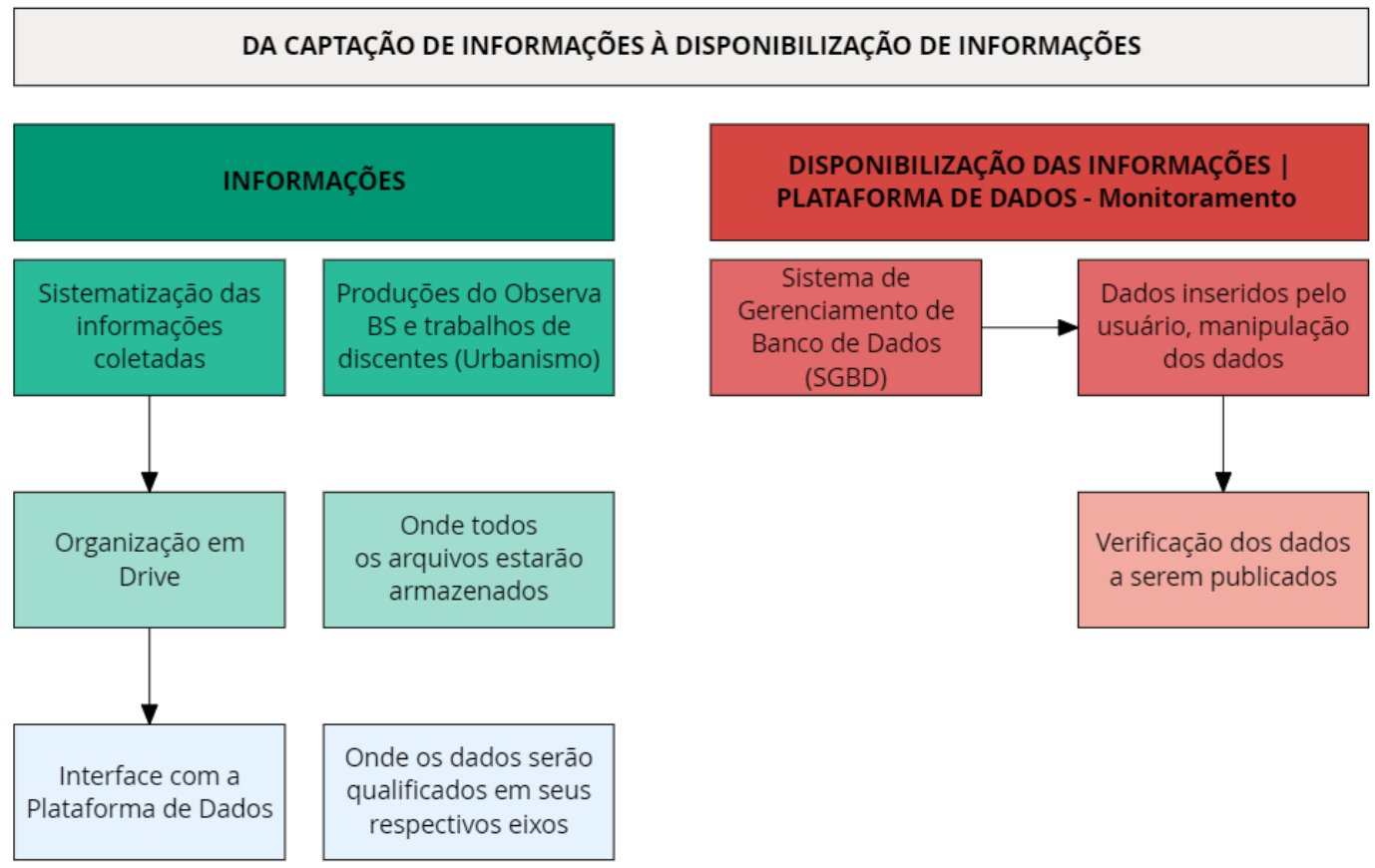

Fonte: Letícia Passarelli Verde (2018)

A primeira e segunda etapas da estruturação da plataforma digital atingiram os resultados esperados, de forma objetiva e satisfatória. Até o momento a plataforma ainda não está disponível aos usuários, pois pretende-se lançá-la primeiramente em caráter experimental e depois oficialmente durante a Jornada de Iniciação Científica, promovida pelo Ipeci, vinculada ao site da UniSantos. A plataforma digital terá continuidade por novos alunos de Iniciação Científica, com apoio de estudantes pesquisadores, integrantes do grupo de pesquisa Observa BS, os quais adequarão a plataforma com base na sua utilização até atingir a sua consolidação.

\section{CONSIDERAÇÕES FINAIS}

A plataforma de dados proposta dará maior alcance na difusão de dados e informações já existentes e produzidas a partir de pesquisas e projetos de extensão do grupo Observa BS e das disciplinas de Urbanismo do curso de Arquitetura e Urbanismo da Unisantos. Almeja-se que a plataforma de dado seja uma ferramenta de pesquisa para ampliar o conhecimento e análises críticas, ao suscitar questionamentos e debates quanto às questões urbanas e metropolitanas nas várias dimensões das premissas da sustentabilidade. Buscar assim, fortalecer a prática da pesquisa, extensão e da cidadania, com transparência, como também fortalecer a participação e o controle social com a democratização do acesso ao à informação qualificada e ao conhecimento.

\section{AGRADECIMENTOS}

Agradecemos ao Conselho Nacional de Desenvolvimento Científico e Tecnológico (CNPq) pelo apoio das bolsas do Programa Institucional de Bolsas de Iniciação Científica (PIBIC), ao Instituto de Estudos, Formação e Assessoria em Políticas Sociais - Instituto Polis, e à Universidade Católica de Santos por proporcionar a articulação do ensino, pesquisa e extensão e por concederem a hospedagem da Plataforma de Dados e Informações do Observatório Socioespacial da Baixada Santista - Observa BS. 


\section{REFERÊNCIAS}

AGEM. Plano Metropolitano de Desenvolvimento Estratégico da Baixada Santista. Disponível em: <http://www.agem.sp.gov.br/midia/pmdebs.pdf.> Acesso em 21 mar. 2018.

CCM. Bancos de dados. Disponível em: <http://br.ccm.net/contents/65-bancos-de-dados>. Acesso em: 01 Nov. 2017.

CHIZZOTTI, Antonio. Metodologia do ensino superior: o ensino com pesquisa. In: CASTANHO, Sérgio; CASTANHO, Maria Eugênia. (Org). Temas e textos em metodologia do Ensino Superior. Campinas: Papirus, 2001. p. 103-112.

JANNUZZI, Paulo de Martino. Consideraçōes sobre o uso, mau uso e abuso dos indicadores sociais na formulação e avaliação de políticas públicas municipais. RAP: Rio de Janeiro, v. 36, n. 1, p. 51-72, jan/fev. 2002.

NEPO, Núcleo de Estudos de População "Elza Berquó". Campinas. Disponível em: <http://www.nepo.unicamp.br/>. Acesso em: 07 dez. 2017.

POLIS, Instituto. Metodologia de Monitoramento, 2015. Disponível em: <http://litoralsustentavel.org.br/indicadores>. Acesso em: 07 dez. 2017.

POLIS, Instituto. Plataforma de Indicadores. Disponível em: <http://instituto.polis.eokoe.com/>. Acesso em: 07 dez. 2017.

PREFEITURA DE SANTOS. Santos. Disponível em: <http://www.santos.sp.gov.br/>. Acesso em: 07 dez. 2017.

PREFEITURA DE SANTOS. SigSantos. Santos. Disponível em:

<https://egov1.santos.sp.gov.br/sigsantosweb/sigsantoswebsd/>. Acesso em: 07 dez. 2017.

PREFEITURA DE SÃO PAULO. GeoSampa. São Paulo. Disponível em: <http://geosampa.prefeitura.sp.gov.br/PaginasPublicas/_SBC.aspx>. Acesso em: 07 dez. 2017.

PROGRAMA CIDADES SUSTENTÁVEIS. Plataforma digital Programa Cidades Sustentáveis. Disponível em: <http://www.cidadessustentaveis.org.br/>. Acesso em: 07 dez. 2017.

REZENDE, Ricardo. Conceitos Fundamentais de Banco de Dados. Rio de Janeiro. Disponível em: <https://www.devmedia.com.br/conceitos-fundamentais-de-banco-de-dados/1649>. Acesso em: 01 Nov. 2017.

RIONOW. Plataforma digital RIONOW. Rio de Janeiro. Disponível em: <http://rionow.org/home.html>. Acesso em: 07 dez. 2017.

SEU ACERVO. Plataforma digital Seu Acervo. Disponível em: <https://sevacervo.com/>. Acesso em: 07 dez. 2018. 